\title{
Variations in the Origin and Course of the Extracranial Vertebral Artery on Multidetector Computed Tomography Angiography
}

\author{
Chia-Ying Lin, ${ }^{1}$ Yi-Sheng Liu, ${ }^{1}$ Ying-Chen Chen, ${ }^{1}$ Yu-Hsiang Shih,,${ }^{1}$ Chao-Chun Chang, ${ }^{2}$ and Ming-Tsung \\ Chuang, ${ }^{1, *}$ \\ ${ }^{1}$ Department of Diagnostic Radiology, National Cheng Kung University Hospital, College of Medicine, National Cheng Kung University, Tainan, Taiwan, R.O.C. \\ ${ }^{2}$ Department of Surgery, National Cheng Kung University Hospital, College of Medicine, National Cheng Kung University, Tainan, Taiwan, R.O.C. \\ "Corresponding author: Ming-Tsung Chuang, Department of Diagnostic Radiology, National Cheng Kung University Hospital, College of Medicine, National Cheng Kung \\ University, No.138, Sheng Li Road, Tainan, 704, Taiwan, R.O.C. Tel: +886-62353535, Fax: +886-62761110, E-mail: u8501122@gmail.com \\ Received 2016 October 30; Revised 2017 September 20; Accepted 2017 October 28.
}

\begin{abstract}
Background: Variations in the origin of vertebral arteries (VA) and its entrance level into the transverse foramen are common and important when planning neck and cervical spine interventions.

Objectives: The aim of this study was to investigate the prevalence of anatomical variations in the origin of vertebral arteries, and anomalous course of the extracranial vertebral artery using dual energy CT angiography.

Patients and Methods: Retrospectively, we reviewed head and neck dual energy computed tomography (DECT) angiographies of 1218 consecutive patients during 2010 - 2014. The images were evaluated with exceptional attention to the origin of VA and its level of entrance into and exit out of the transverse foramen of the cervical spine, specific variations of VA including fenestration and intradural course of $\mathrm{C} 1$ - C2 VA.

Results: We found a rare case of double origin of left VA with bifid origins (one from the aortic arch and the other from the left subclavian artery) and entering the transverse foramen at C6 and C5 levels. The majority of left VA (1173/1219, 96.2\%) originated from the left subclavian artery. Most of them (1159/1173, 98.8\%) entered the C6 transverse foramen. Forty - six of left VA (46/1219, 3.8\%) originated from the aortic arch and thirty - seven (80.4\%) of them entered the $\mathrm{C} 5$ transverse foramen. When the left VA arose from the aorta, there was a significant higher rate of anomalous entrance level (C4 or C5) to normal C6 entrance level $(\mathrm{P}<0.001)$. The majority of right VA $(1209 / 1218,99.3 \%)$ originated from the right subclavian artery. Eight $(0.7 \%)$ of right VA arose from the aberrant right subclavian artery (ARSA). One rare case of right VA from right common carotid artery (CCA) entered the transverse foramen at C4 level with coexisting ARSA. Five cases had atlanto - axial fenestration of the VAs and five cases had intradural course of left C1 - C2 VAs.

Conclusion: The study showed anatomical variations of the VA in its origin and extracranial course. A pre-operative CT angiography is useful to identify the anomalous extracranial VA course. It reduces the risk of intra - procedure VA injury.
\end{abstract}

Keywords: Vertebral Artery, Neck, Computed Tomography Angiography, Anatomy

\section{Background}

The vertebral artery (VA) is divided into four segments classically. The first three segments are extracranial and the last is intracranial. The first segment (preforaminal, V1) starts from the dorsal aspect of the subclavian artery to the C6 transverse process. The second segment (foraminal, V2) begins from the sixth cervical vertebra to the second cervical vertebra passing through respective transverse foramina at each vertebral level. The third segment (atlantic, V3) starts from $\mathrm{C} 2$ to the foramen magnum. Finally, the fourth segment (intradural, V4) courses from the foramen magnum to the vertebrobasilar junction. Normally, the VA en- ters C6 transverse foramen and exits the transverse foramen at $\mathrm{C} 2$ level. Previous publications report the incidence of anomalous extracranial VA course is about $6.3 \%$ of cases (1).

Variations in the origin of VAs and its entrance level of entry into the transverse foramen are common (2). They are usually found incidentally without relevant clinical symptoms (3). However, these important variations should be noticed when planning surgical or radiological interventions to the neck and cervical spine and should not be injured during the procedures $(4,5)$. 


\section{Objectives}

The aim of this study was to investigate the incidence of anatomical variations in the origin of VAs, and their relationship to the anomalous course of the extracranial VA relevant to the subaxial cervical spine with dual energy computed tomography (DECT) angiography.

\section{Patients and Methods}

\subsection{Patients}

The retrospective study was approved by the institutional review board of the hospital. A total of 1291 patients who underwent head and neck dual energy multidetector computed tomography (MDCT) angiography between September 2010 and December 2014 were evaluated. Seventy - three patients were excluded for the following reasons: inadequate image quality $(n=3)$; near total occlusion or agenesis of the VA $(n=3)$; prior aortic surgery ( $n$ $=1$ ); inadequate scan coverage of the head and neck region $(\mathrm{n}=10)$; missing demographic data $(\mathrm{n}=1)$; same subject underwent repetitive computed tomographic angiography (CTA) examination $(n=55)$. Finally, 1218 patients (652 male and 566 female; mean age, $56.78 \pm 17.24$ years; age range, 0 - 93 years) were enrolled in our study. The indications for CT angiography (Table 1) were stroke survey (including carotid stenosis), intracranial hemorrhage (including subarachnoid hemorrhage, subdural hemorrhage, epidural hemorrhage, and parenchymal hemorrhage), headache, pre-operative evaluation (including cervical spine trauma, vascular tumor), suspected vascular lesions (e.g. arteriovenous malformation, carotid - cavernous fistula), and other indications (e.g. venous thrombosis, head and neck bleeding).

\begin{tabular}{lc}
\hline Table 1. Indications for CT Angiography & \\
\hline Indications & Percentage \\
\hline Stroke survey & $34.6 \%(422 / 1218)$ \\
\hline Intracranial hemorrhage & $28.3 \%(345 / 1218)$ \\
\hline Headache & $28.3 \%(345 / 1218)$ \\
\hline Pre- operative evaluation & $3.4 \%(42 / 1218)$ \\
\hline Suspected vascular lesions & $3.0 \%(37 / 1218)$ \\
\hline Others & $2.2 \%(27 / 1218)$ \\
\hline
\end{tabular}

\subsection{CT Image Acquisition, Postprocessing, and Analysis}

All CT examinations were performed with a second generation, dual - energy multidetector CT scanner (somatom definition flash CT; siemens healthcare, Forchheim, Germany). Both precontrast and postcontrast scans were obtained in dual - energy mode. Two different tube voltages and currents were used at the same time: tube A at $100 \mathrm{kV}$ and $104 \mathrm{mAs}$ and tube B at $140 \mathrm{kV}$ and $104 \mathrm{mAs}$. For each diagnostic scan, $60 \mathrm{ml}$ of iodinated contrast medium was intravenously administered via a power injector at a rate of $4 \mathrm{~mL} / \mathrm{s}$ and followed by $35 \mathrm{~mL}$ - saline chase. The CT scan was triggered using a bolus - tracking technique with the region of interest (ROI) placed in the ascending aorta and the image acquisition started 2 seconds once the attenuation reached the predefined threshold (100 Hounsfield units). Data were acquired from the aortic arch to the vertex.

Postcontrast images were reconstructed with 1 - $\mathrm{mm}$ thickness/interval and transferred to the workstation (AZE virtual place version 3.2; AZE Co Ltd, Tokyo, Japan) for further processing. The noncontrast CT, source images from CTA, reformatted coronal and sagittal images, curved planar reformatted images, and volume - rendered images were transferred to the picture archiving and communication system (PACS) for image interpretation and comparison.

The images were evaluated with focus on the origin of VA and its level of entrance into and exit out of the transverse foramen, and specific variations of VA including fenestration and intradural course of C1 - C2 VA. Two staff neuroradiologists with 11 and 9 years of experience reviewed the images, with final decisions made by consensus.

\subsection{Statistical Analysis}

Categorical data were presented as number (percentages), and non - categorical data as the mean \pm standard deviation. Comparison of categorical variables between the study groups was performed using Chi square test. Fisher's exact test was used instead when the expected frequency was less than 5. For all comparisons, a P value < 0.05 was considered significant. All statistical computations were performed by using the SPSS 17.0 statistics software (SPSS Inc., Chicago, IL, USA).

\section{Results}

Table 2 shows the origins and courses of the left VA. A rare case of double origin of left VA was observed in our study (Figure 1). The bifid origins of left VA arose from the left subclavian artery, and aortic arch respectively and unite to form the VA. The VA enters the transverse foramen at $\mathrm{C} 6$ and $\mathrm{C} 5$ levels separately.

The majority of left VA(1173/1219, 96.2\%) originated from the left subclavian artery. Most of them entered the transverse foramen at C6 level (1159/1173, 98.8\%), others entered the transverse foramen at C4 or C5 level (14/1173,1.2\%). All of the left VA exited at $\mathrm{C} 2$ level. 


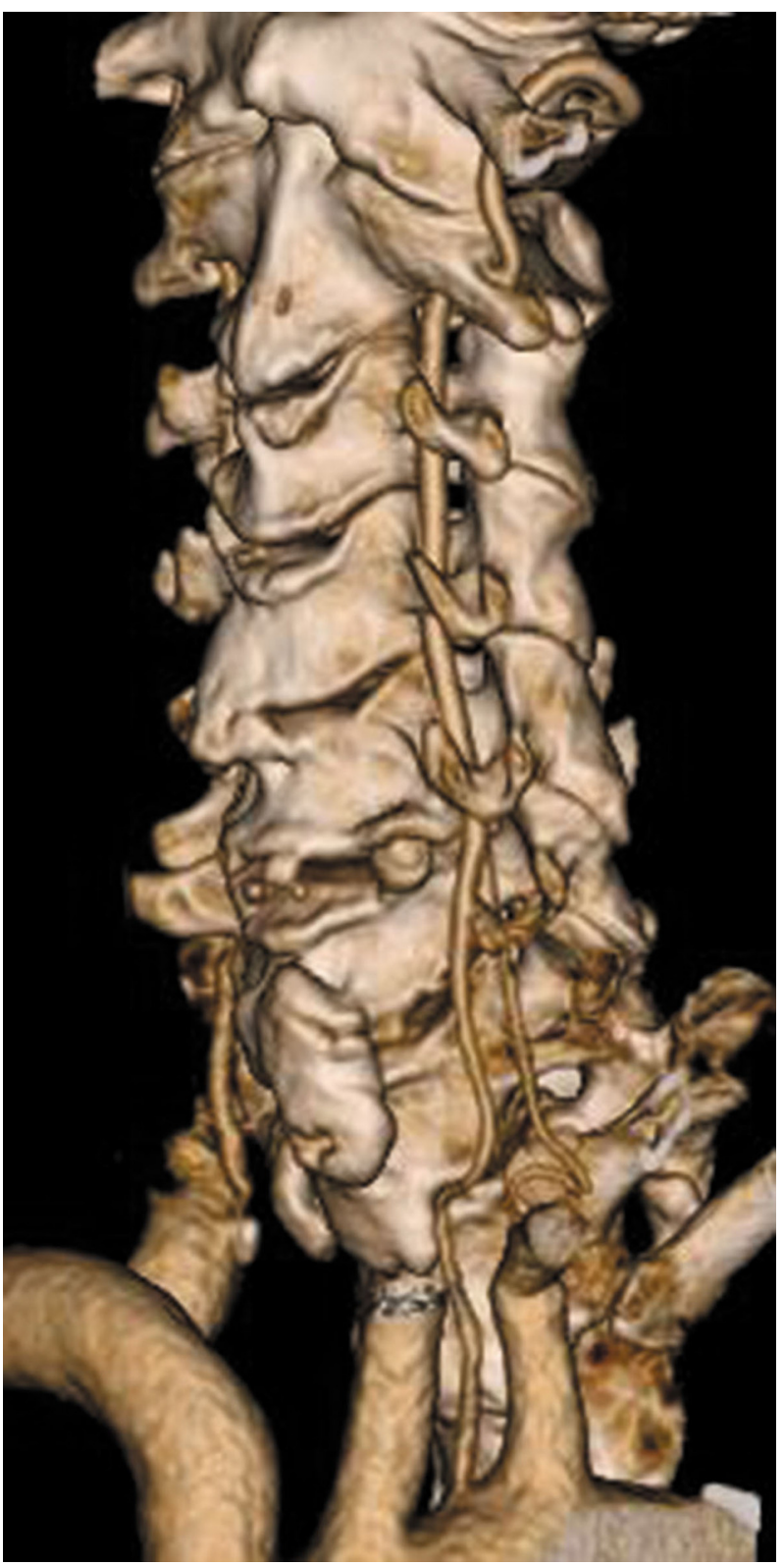

Figure 1. A 62 - year - old female with ischemic stroke underwent CT angiography. The three dimensional (3D) volume-rendering (VR) computed tomographic angiography (CTA) image demonstrates double origins of the left vertebral artery (VA) from the left subclavian artery and aortic arch.

Forty-six left VA(3.8\%) originated directly from the aortic arch. Seven of them entered the transverse foramen at the C4 level (15.2\%), thirty - seven at C5 level (80.4\%), and two of them at C6 level (4.3\%).

When the left VA arose from the aorta, there was a statistically significant higher rate of anomalous entrance level (C4 or C5) to normal entrance level (C6) $(\mathrm{P}<0.001$
Table 2. Results of Variation in the Origin of Left Vertebral Artery and Its Level of Entrance into the Transverse Foramen ${ }^{\mathrm{a}, \mathrm{b}}$

\begin{tabular}{|c|c|c|}
\hline \multirow{2}{*}{$\begin{array}{l}\text { Entrance into Left Transverse } \\
\text { Foramen }\end{array}$} & \multicolumn{2}{|c|}{ Origin of Left VA } \\
\hline & Aorta & Left subclavian artery \\
\hline $\mathbf{I V}+\mathbf{V}$ & $44 / 46(95.7 \%)$ & $14 / 1173(1.2 \%)$ \\
\hline VI & $2 / 46(4.3 \%)$ & $1159 / 1173(98.8 \%)$ \\
\hline
\end{tabular}

[Fisher exact test]; odds ratio, 1821.3; 95\% confidence interval [CI], 401.59 - 8259.87).

Table 3 shows the origins and courses of the right VA. The majority of right VA $(1209 / 1218,99.3 \%)$ originated from the right subclavian artery, eight of the right VA (0.7 \%) ascended from the aberrant right subclavian artery (ARSA), and one right VA $(0.1 \%)$ started from the common carotid artery (CCA). All of the right VAs exited at C2 level.

Table 3. Results of Variation in the Origin of Right VA and Its Level of Entrance into the Transverse Foramen ${ }^{\mathrm{a}}$

\begin{tabular}{lcc}
\hline $\begin{array}{l}\text { Entrance into Right } \\
\text { Transverse Foramen }\end{array}$ & \multicolumn{2}{c}{ Origin of Right VA } \\
\cline { 2 - 3 } & ARSA and right CCA & $\begin{array}{c}\text { Right subclavian } \\
\text { artery }\end{array}$ \\
\hline IV + V & $1 / 9(11.1 \%)$ & $47 / 1209(3.9 \%)$ \\
VI & $8 / 9(88.9 \%)$ & $1162 / 1209(96.1 \%)$ \\
\hline $\begin{array}{l}\text { Abbreviations: ARSA, aberrant right subclavian artery; CCA, common carotid } \\
\text { artery; VA, vertebral artery. } \\
\text { a Data show the number of patients, with percentages in parentheses. }\end{array}$
\end{tabular}

About 96.1\% (1162/1209) of right VAs arising from right subclavian artery entered the transverse foramen at C6. All right VAs from aberrant right subclavian artery (ARSA) entered the transverse foramen at C6 level. The right VA arose from the right subclavian artery inserted the transverse foramen between $\mathrm{C} 4$ and $\mathrm{C} 6$ levels. A rare case of right VA from the right CCA entered the transverse foramen at $\mathrm{C} 4$ level with coexisting ARSA (Figure 2).

All right VA arising from the ARSA entered the transverse foramen at $\mathrm{C} 6$ level. In contrary to the left side, there is no significant higher rate of anomalous right VA entrance level ( $\mathrm{C} 4$ and $\mathrm{C} 5$ ) when there is an unusual origin of the right VA(including ARSA or right CCA) $(\mathrm{P}=0.304$ [Fisher exact test]; odds ratio, 3.090; 95\% confidence interval [CI], 0.38 - 25.28).

In the study, there is relationship between variations of VA entrance level on both sides. If anomalous VA entrance level (C4 or C5) is found on one side, there is a significant higher rate of variation of that on the contralateral side 

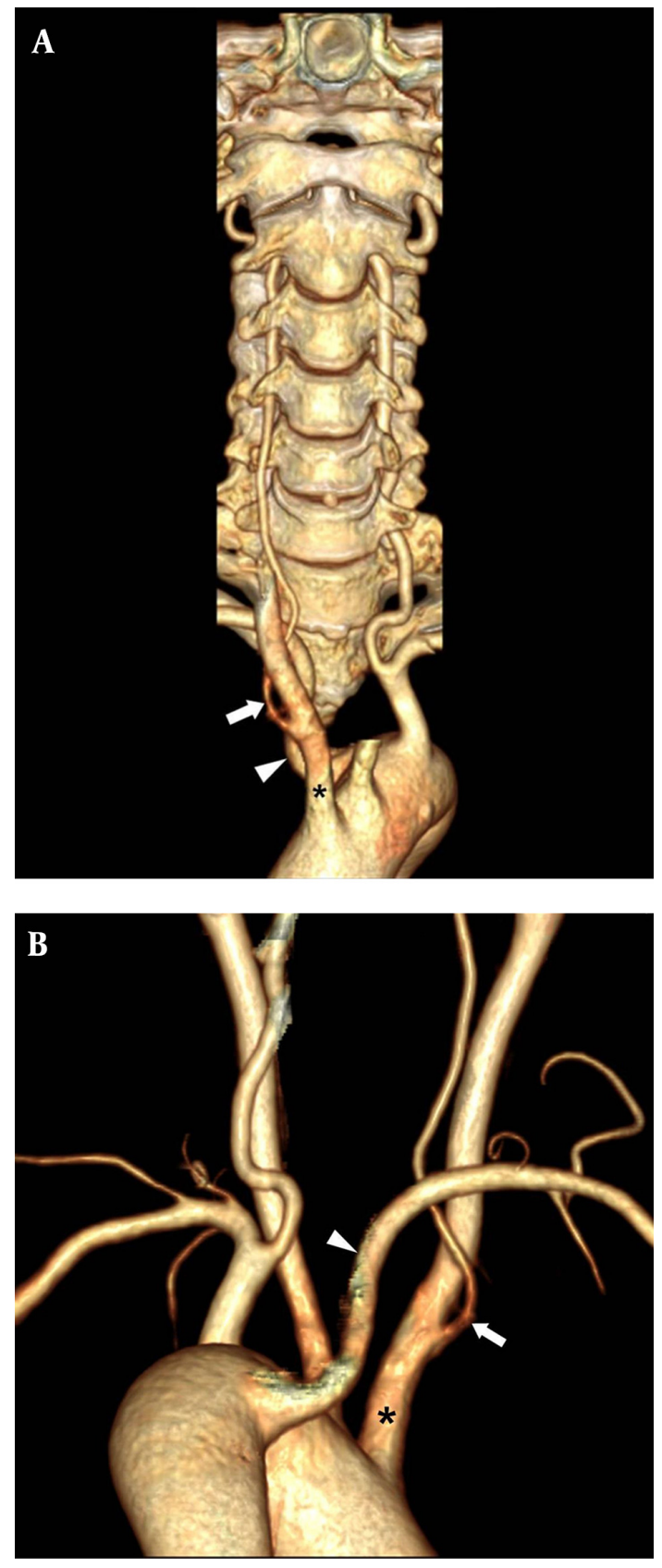

Figure 2. The three dimensional volume - rendering computed tomographic angiography vertebral artery (3D VR CTA) images of neck viewed anteriorly (A) and posteriorly (B) demonstrate a rare variation of anomalous origin of the right vertebral artery (VA) (arrow) arising from the right common carotid artery (CCA) (asterisk) and entering the transverse foramen at $\mathrm{C} 4$ level with coexisting aberrant right subclavian artery (ARSA) (arrowhead).

( $\mathrm{P}<0.001$ [Fisher exact test]; odds ratio, 9.286; 95\% confidence interval [CI], $4.60-18.75)$. However, there is no significant correlation between the VA origins on both sides. If anomalous VA origin (e.g. right VA from ARSA, right CCA; or left VA from aorta) is found on one side, there is no significant higher rate of variation of that on the contralateral side ( $\mathrm{P}=0.294$ [Fisher exact test]; odds ratio, 3.233; 95\% confidence interval [CI], 0.40 - 26.41).

\subsection{VA Fenestration}

There are two cases of atlanto - axial fenestration of the right VA and three cases of atlanto - axial fenestration of the left VA found in our study. All of the VAs arose from the subclavian artery.

\subsection{Intradural Course of the C1 - C2 VA}

Five cases of intradural course of the left C1 - C2 VA were observed in our study. In these cases, the VA coursed inferiorly and turned medially beneath the $\mathrm{C} 1$ posterior arch before piercing the dura instead of traversing the $\mathrm{C} 1$ foramen.

\section{Discussion}

A brief review of the embryology of the aortic arch and great vessels (6) makes understanding the variations easy. The thoracic aorta is derived from the embryonic primitive ventral and dorsal aortae that evolve into the external and internal carotid arteries. In the cervical region, the postcostal longitudinal anastomosis between the $\mathrm{C} 1$ and $\mathrm{C} 7 \mathrm{seg}$ mental arteries form the VA. The bilateral VAs normally develop from the distal part of the 7th dorsal intersegmental arteries. The left 7 th dorsal intersegmental artery remains to form the proximal left subclavian artery to the level of the internal thoracic artery, while the right 7th dorsal intersegmental artery builds the distal one third of the proximal right subclavian artery (Figure 3). Anatomical variations arise from persistence or obliteration of these various arches. Anomalous origins of VA occur when there is aberrant anastomosis occurs during the embryonic development of the arch. The site of the anastomosis will define the eventual unusual origin. In the neck, three major extraspinal longitudinal intersegmental anastomoses between the $\mathrm{C} 1$ and $\mathrm{C} 7$ are formed by the VA, ascending cervical artery, and deep cervical artery. The VA lies within the transverse process; the ascending cervical artery lies ventral to the transverse process; and the deep cervical artery lies dorsal to the transverse process. The difference in the entry level of the VA is related to the dominance of a ventral or dorsal extraspinal group of longitudinal intersegmental anastomosis. For example, the VA going into the transverse foramen at $\mathrm{C} 5, \mathrm{C} 4, \mathrm{C} 3$ level stands for the ascending cervical artery which is not depicted as a distinct vessel in these variations (Figure 4) (7). 


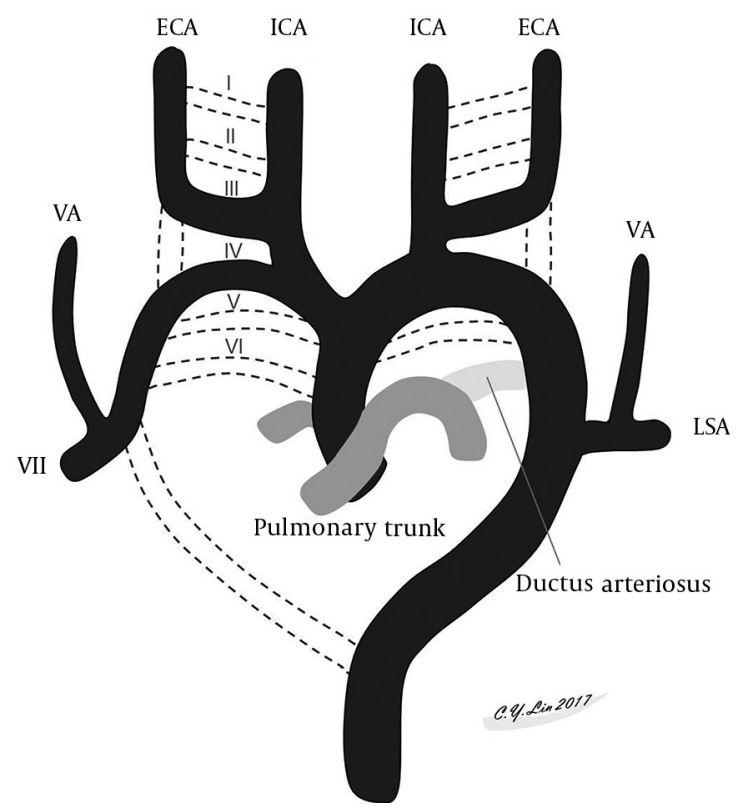

Figure 3. Schematic presentation of the embryological development of the aortic arch, its branches and vertebral arteries (VAs). Six paired aortic arches connect paired ventral aortae and dorsal aortae. The I, II, V aortic arches regress. The third, fourth, and sixth aortic arches grow and develop into arteries. The fourth aortic arches progress into the aortic arch. The ventral bud of the sixth aortic arch evolves into the pulmonary artery. Portions of the third aortic arch and paired ventral aortae and dorsal aortae develop into the external and internal carotid arteries. The seventh intersegmental arteries migrate cranially and form the subclavian arteries. Abbreviations: ECA, external carotid artery; ICA, internal carotid artery; LSA, left subclavian artery; VA, vertebral artery.

A complete understanding of anomalous VAs is important when doing both diagnostic and interventional angiography (4). One may misinterpreted as absent VA if the VA is not recognized in their usual position. Identifying the abnormal level of VA entrance is crucial prior to the operation, especially with high level of entrance due to lack of bony protection. For example, during cervical spine surgery, one may injury the VA with high level of entrance when dissecting the anterior branch of the transverse process and the longus colli muscle with unawareness and overconfidence. The abnormal course of extradural VA can also complicate the corpectomy foraminotomy, and percutaneous nerve block, causing arterial laceration or pseudoaneurysm formation $(5,8,9)$. Therefore, it is important to perform appropriate imaging study to delineate the extradural VA course preoperatively and prevent iatrogenic injury.

CT angiography is principal imaging modality for assessment of vascular anomalies nowadays due to high spatial resolution, short acquisition time, and widespread availability. In recent years, DECT has an added value in the evaluation of vascular structures (10). DECT offers tissue differentiation, characterization (i.e., hide or highlight calcified plaques, differentiation between hemorrhage and contrast after previous contrast injection), artifacts reduction (i.e., non - threshold dependent automated bone removal), contrast - to - noise ratio (CNR) and signal - to noise ratio (SNR) remodeling. DECT can differentiate high - density lesions, such as iodine or hemorrhage based on the change in $x$ - ray attenuation (10-13). Therefore, we use DECT as a non - invasive and reliable tool to diagnose vascular anomalies in the head and neck region.

The present study depicts the frequency and imaging findings of the common variations in the origin and extracranial course of VAs on the DECT from a single medical center in Southern Taiwan and contributes data from an Asian patient group to the current knowledge.

In comparison with the previous study (Tables 4 and 5), Ranganatha et al. (14) studied 19 formalin fixed adult cadavers. Meila et al. (1) reviewed CTA imaging of VA of 539 patients using a 16 - detector row CT scanner with particular focus on the origin and the course of the VA. Uchino et al. (2) surveyed CT angiographic images of 2,287 Japanese patients acquired using 64 - slice multidetector CT scanners from the aortic arch to the intracranial part, most had or were presumed of having cerebrovascular diseases. Shin et al. (15) observed 16 - row multi - detector CT data of 460 Korean patients about the origin and course of VA, in addition to the level of VA entrance into the cervical transverse foramen. Hong et al. (16) reviewed 350 cases of VAs on three - dimensional CT angiographies. Measurements between the surgical landmarks to the extraosseous portions of the VA and the transverse foraminal diameter were done.

The VA usually originates from the subclavian artery and enters the sixth cervical transverse foramen between the scalenus anterior muscle and longus colli muscle. Lasjaunias et al. (17) reported extradural variations of VA in the vertebral canal from 66 to foramen magnum. They stated that the ascending cervical flow to distal VA could cause the C5 to C3 vertebral entry of VA.

In more than $90 \%$ of the cases, the VA originated from the subclavian artery and went into the C6 transverse foramen. Therefore, in most of the cases, the VA corresponds to the $\mathrm{C} 6$ metamere. The second most common variation was the left VA arising directly from the aortic arch, with an incidence rate of $3.7 \%$ in our study in comparison to 3.8\%, 6.3\% and 6.6\% reported in Celikyay (18), Melia (1), and Bronx (19) series, respectively. Shin et al. (15) showed that the transverse foramen of the 6 th cervical vertebra was the most common site of VA entry (left side, $92 \%, n=421$; right side, $94.3 \%, \mathrm{n}=434)$. Other anomalous courses reported including the VAs entered into the $\mathrm{C} 7$ transverse foramen (left side, $0.4 \%, \mathrm{n}=2$ ), $\mathrm{C} 5$ transverse foramen (left side, $6.5 \%$, $\mathrm{n}=30$; right side, $3.3 \%, \mathrm{n}=15$ ), and $\mathrm{C} 4$ transverse foramen 
A

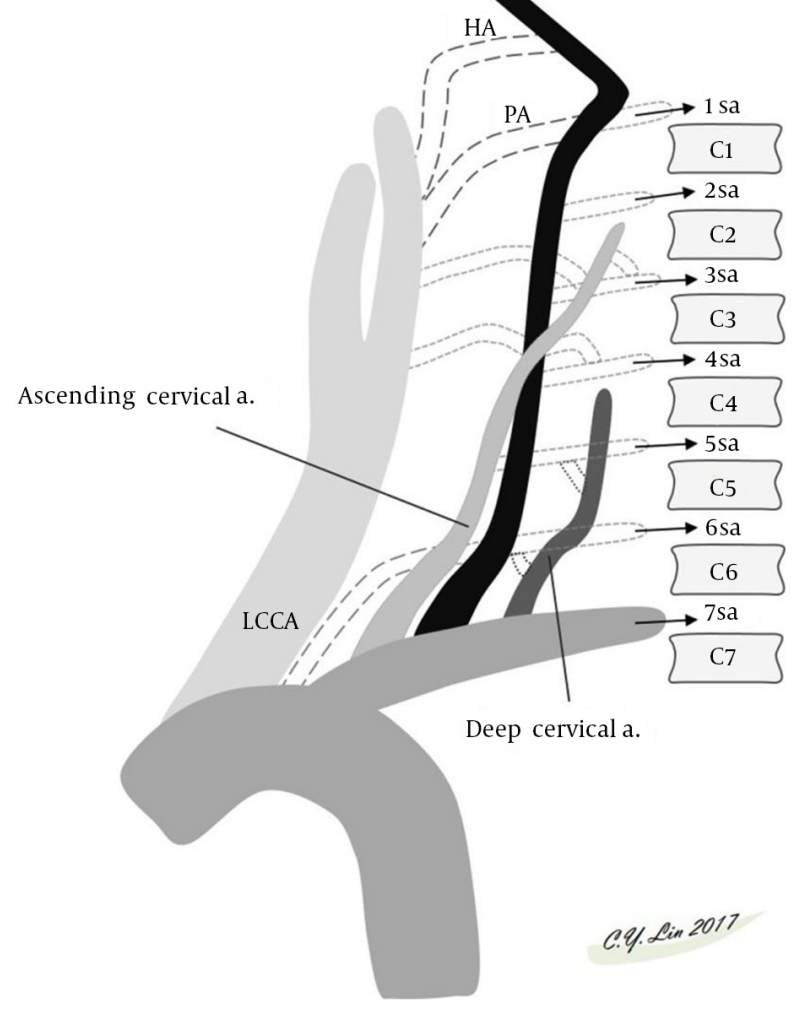

B

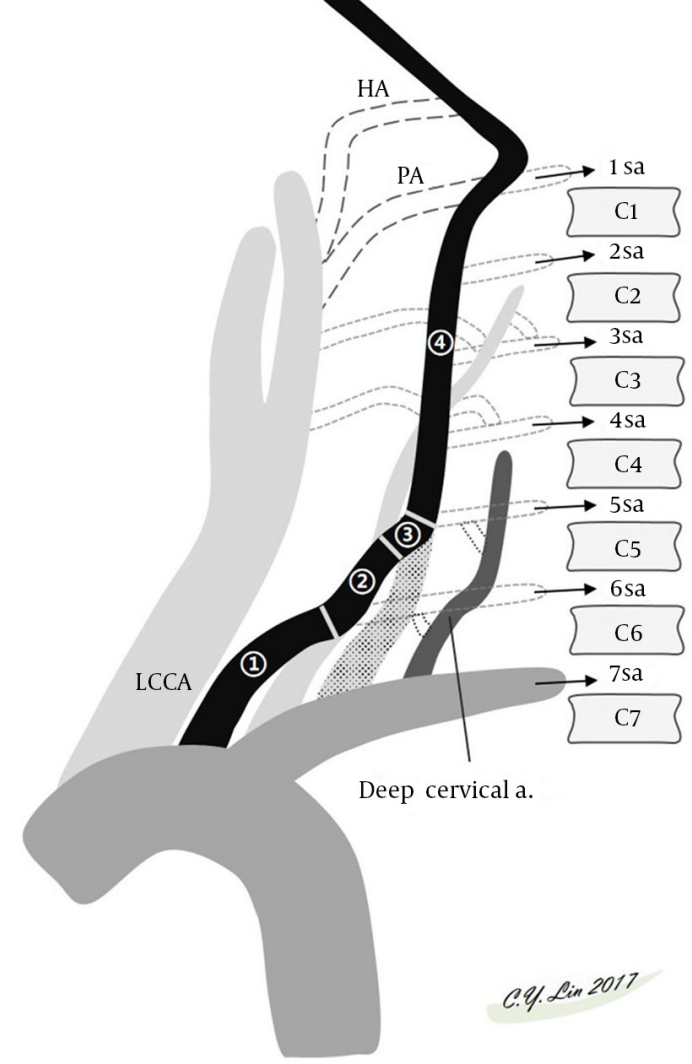

Figure 4. Schematic presentation of embryological development of normal VA (A) and common variation of left VA starting directly from the aortic arch (B). A, Normally, the VA formed by longitudinal intersegmental anastomosis between the $\mathrm{C} 1$ and $\mathrm{C} 7$ segmental arteries lies within the transverse foramen. Two other major cervical accessory paramedian intersegmental anastomoses (the ventral ascending cervical artery and the dorsal deep cervical artery) lie parallel to the VA. The difference in the entry level of the VA is related to dominance of a ventral or dorsal extraspinal group of longitudinal intersegmental anastomosis. B, The left VA is formed by persistent sixth cervical segmental artery (1) proximally, connecting to the corresponding ascending cervical artery (2), the 5th segmental artery (3) entering C5 transverse foramen, and following normal foraminal VA course (4) distally. In this variation, the left VA will enter the transverse foramen at C5 level, while the ascending cervical artery will not be depicted as a separate vessel. Abbreviations: HA, hypoglossal artery; LCCA, left common carotid artery; PA, proatlantal artery; sa, segmental artery; VA, vertebral artery. 


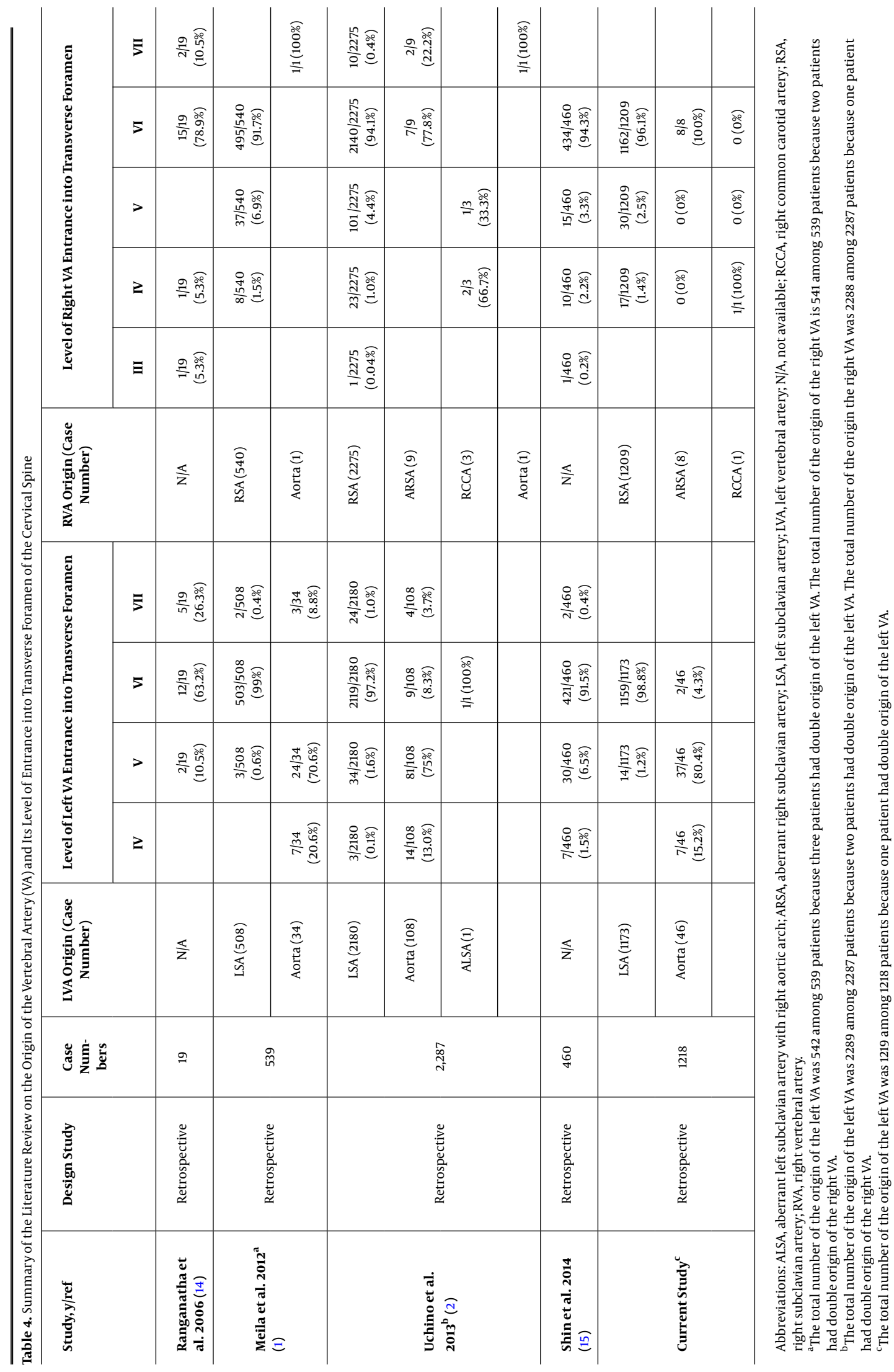


(left side, $1.5 \%, \mathrm{n}=7$; right side, $2.2 \%, \mathrm{n}=10$ ). Uchino et al. (2) showed that the VA originated from the aorta between the left CCA and left subclavian artery in 94 patients (4.1\%). The right VA originated from the extreme proximal segment of the right subclavian artery in 72 patients (3.1\%), from ARSA in nine patients $(0.4 \%)$, and from the right CCA in one patient. The majority of the VA entered the sixth transverse foramen (left side, 93.0\%, $\mathrm{n}=2127$; right side, $93.8 \%, \mathrm{n}=$ 2146).

Anomalous origin and anomalous entry level into the transverse foramen correlated strongly. Hong et al. (16) showed that abnormal entrance level was observed in $5.1 \%$ of the specimens (36 VA course), with the entrance level at $\mathrm{C} 4$, C5, or C7 transverse foramen in $1.6 \%, 3.3 \%$, and $0.3 \%$, respectively. However, the study did not take the origin of the VA into consideration. Meila et al. (1) reported that a left VA originating directly from the aortic arch will not enter the transverse foramen at $\mathrm{C} 6$ level but instead at a higher at $\mathrm{C} 4$ (1.3\%) or C5 (4.5\%) level when the origin is proximal to the subclavian artery, or at C7 (0.56\%) with an aortic arch origin distal to the subclavian artery.

Our results showed the majority of left VA originated from the left subclavian artery (96.2\%) and 3.8\% of the left VA originated directly from the aortic arch, which were similar to the results from the study conducted by Uchino et al. (2). The independent origin of the left VA from the aortic arch is associated with a significant higher incidence of the anomalous course of VA entrance into the transverse foramen (C4 or C5), which is similar to the result from the study carried out by Meila et al. (1).

In our study, the relationship between variations in both VAs entrance level is well demonstrated. If ipsilateral anomalous VA entrance level is found, there is a significant higher rate of that variation on the contralateral side. Variations of the VA are usually asymptomatic and are found incidentally during radiology practice (3). Komiyana et al. (20) mentioned a significantly higher incidence of VA dissection when the left VA came directly from the aortic arch as compared to VA of the subclavian artery origin. However, no such correlation is observed in our study. One rare case of right VA from the right CCA with coexisting ARSA was found in our study. This variation has been reported in the previous study (21). A possible explanation is persistent lower (third through sixth) cervical intersegmental arteries and an involution of the ipsilateral middle dorsal aorta, between the seventh cervical intersegmental artery and the persistent cervical intersegmental artery, resulting in abnormal origin of the VA from the CCA with ipsilateral aberrant subclavian artery (Figure 5). In clinical practice, ligation of the CCA may compromise the blood supply to the posterior fossa and hence cause ischemic stroke.

Duan et al. (22) used 3D images from head and neck

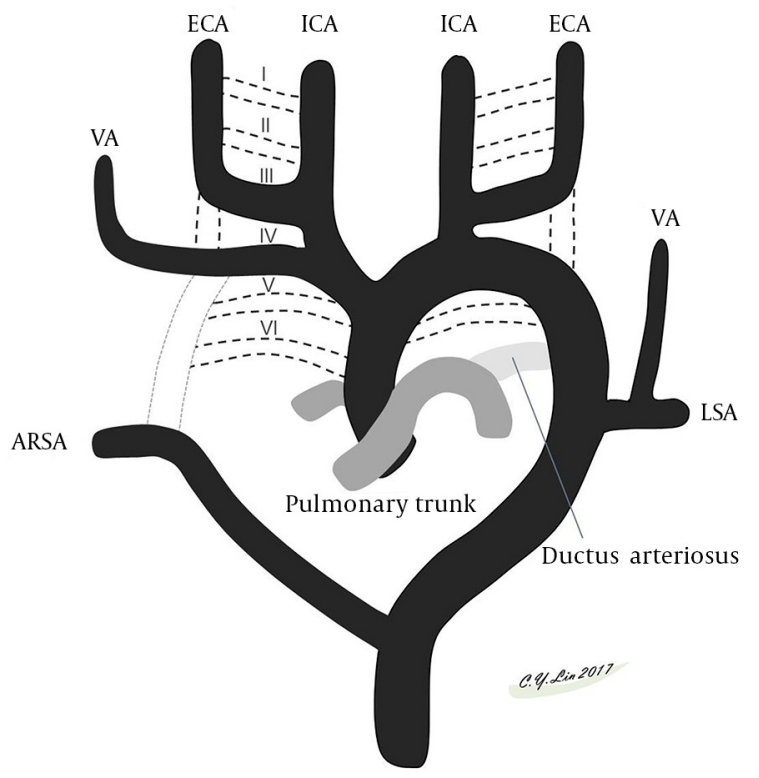

Figure 5. Schematic presentation of embryological development of anomalous origin of right vertebral artery (VA) arising from right common carotid artery (CCA) with coexisting aberrant right subclavian artery (ARSA). In this variation, the origin of right VA is developed from persistent fourth cervical segmental artery and regression of the ipsilateral middle dorsal aorta, resulting in abnormal origin of the VA from the CCA with coexisting ARSA. Abbreviations: ECA, external carotid artery; ICA, internal carotid artery; LSA, left subclavian artery; VA, vertebral artery.

CTA to observe the courses and branch of V3 - V4 segment and their peripheral venous vascular plexus. Variations were observed in thirteen percent (13/98) of the patients including not going through the transverse foramen of atlas, multi - branches of V4 or fenestrated V3, and small size $(<1 \mathrm{~mm})$ in seven patients. In our study, fenestration in V3 segment of VA was found in the right VA $(n=2)$ and left VA $(n=3)$ in the atlantoaxial region. All of them arose from the subclavian artery. Failure to regress of the two intersegmental arteries is the proposed embryological explanation. Several authors have demonstrated that cerebral fenestrations are predisposed to form aneurysm (23). The hypothesis is that the collagen defects in the tunica media will weaken the arterial wall at the edges of the fenestration. However, some said that fenestration - associated aneurysms are incidental, and that the incidence of fenestration - related aneurysm is the same as the overall population (24). In our study, there was no significant correlation between anomalous VA origin and fenestration (Fisher exact test, $\mathrm{P}=1.000$ ). There was no dissection or aneurysm of VA observed when VA fenestration was present.

There were five patients with intradural course of $\mathrm{C} 1$ - C2 VA in our study. Intradural course of the C1 - C2 VA 


\begin{tabular}{|c|c|c|c|c|c|c|c|c|c|}
\hline \multirow{2}{*}{$\begin{array}{l}\text { Study, } \\
\text { y/ref }\end{array}$} & \multirow[t]{2}{*}{ Case No. } & \multirow{2}{*}{$\begin{array}{c}\text { LVA } \\
\text { origin }\end{array}$} & \multicolumn{3}{|c|}{ Level of left VA entrance into transverse foramen } & \multirow{2}{*}{$\begin{array}{c}\text { RVA } \\
\text { origin }\end{array}$} & \multicolumn{3}{|c|}{ Level of right VA entrance into transverse foramen } \\
\hline & & & IV & $\mathrm{V}$ & VI & & IV & $\mathrm{V}$ & VI \\
\hline \multirow{3}{*}{$\begin{array}{l}\text { Uchino } \\
\text { et al. } \\
2013 \\
(2)\end{array}$} & 1 & Aorta LSA & & 1 & 1 & & & & \\
\hline & 2 & Aorta LSA & & 1 & 1 & & & & \\
\hline & 3 & & & & & RSA RSA & & 1 & 1 \\
\hline \multirow{3}{*}{$\begin{array}{l}\text { Meila } \\
\text { et al. } \\
2012 \\
(1)\end{array}$} & 2 & Aorta LSA & 1 & & 1 & & & & \\
\hline & $\begin{array}{c}3 \\
\text { (Bilateral) }\end{array}$ & $\begin{array}{l}\text { Aorta } \\
\text { Aorta }\end{array}$ & 1 & 1 & & RSA RSA & 1 & & 1 \\
\hline & 4 & Aorta LSA & & 1 & 1 & & & & \\
\hline $\begin{array}{l}\text { Current } \\
\text { study }\end{array}$ & 1 & Aorta LSA & & 1 & 1 & & & & \\
\hline
\end{tabular}

Abbreviations: ALSA, aberrant left subclavian artery with right aortic arch; ARSA, aberrant right subclavian artery; LSA, left subclavian artery; LVA, left vertebral artery; $\mathrm{N} / \mathrm{A}$, not available; RCCA, right common carotid artery; RSA, right subclavian artery; RVA, right vertebral artery.

means the VA course posteromedially after exiting the $\mathrm{C} 2$ transverse foramen and pierce the spinal canal between $\mathrm{C} 1$ and $\mathrm{C} 2$, not traversing through the $\mathrm{C} 1$ transverse foramen. The embryological explanation is the first intersegmental artery remains without persistence of the first VA. Congenital osseous anomalies, such as hypoplastic odontoid, os odontoideum, and bifid $\mathrm{C} 1$ posterior arch have been reported to increase the incidence of intradural course of the C1-C2 VA(25). However, in this study, we did not observe the osseous anomalies in the patient with intradural course of the $\mathrm{C} 1$ - C2 VA.

Preoperative scan to identify the anomalies of the vertebral artery (such as anomalous entry and exit level, or the artery being too medial) can help to minimize the complications during cervical spine surgery, especially cervical corpectomy, fusion, and vertebral restoration. Limitations of the study are as follows. There was a selection bias due to its retrospective nature. The sample of the patients was relatively small. Despite the limitations of the study, the study provides useful anatomical data about vertebral artery in Asian people.

In conclusion, the present study showed common anatomical variations in the origin of vertebral arteries, and their relationship to the anomalous course of the vertebral artery in the cervical spine. These variations are crucial when performing diagnostic angiography, endovascular interventions or cervical spine surgery. A thorough pre - operative CT angiography is useful to identify the anomalous extracranial VA course. Appreciation of these entities will reduce the risk of VA injury during procedure and aid in patient care.

\section{Acknowledgments}

This study was funded by a grant from National Cheng Kung university hospital (NCKUH - 10305011).

\section{Footnotes}

Conflict of interest: The authors declare that they have no conflicts of interest.

Ethical Statement: This article does not contain any studies with human participants or animals performed by any of the authors.

Financial Disclosure: The authors have nothing to disclose.

\section{References}

1. Meila D, Tysiac M, Petersen M, Theisen O, Wetter A, Mangold A, et al. Origin and course of the extracranial vertebral artery: CTA findings and embryologic considerations. Clin Neuroradiol. 2012;22(4):327-33. doi: 10.1007/s00062-012-0171-0. [PubMed: 22941252].

2. Uchino A, Saito N, Takahashi M, Okada Y, Kozawa E, Nishi N, et al. Variations in the origin of the vertebral artery and its level of entry into the transverse foramen diagnosed by CT angiography. Neuroradiology. 2013;55(5):585-94. doi: 10.1007/s00234-013-1142-0. [PubMed: 23344682].

3. Lale P, Toprak U, Yagız G, Kaya T, Uyanık SA. Variations in the branching pattern of the aortic arch detected with computerized tomography angiography. Adv Radiol. 2014;2014.

4. Satti SR, Cerniglia CA, Koenigsberg RA. Cervical vertebral artery variations: an anatomic study. AJNR Am J Neuroradiol. 2007;28(5):976-80. [PubMed: 17494682].

5. Burke JP, Gerszten PC, Welch WC. Iatrogenic vertebral artery injury during anterior cervical spine surgery. Spine J. 2005;5(5):508-14. doi: 10.1016/j.spinee.2004.11.015. [PubMed: 16153577] discussion 514.

6. Congdon ED. Transformation of the aortic arch system during the development of the human embryo. Contrib Embryol. 1922;14(9):47-110.

7. George B, Bruneau M. Pathology and surgery around the vertebral artery. Paris: Springer; 2011. pp. 5-24.Embryology of the vertebral artery.

8. Inamasu J, Guiot BH. Iatrogenic vertebral artery injury. Acta Neurol Scand. 2005;112(6):349-57. doi: 10.1111/j.1600-0404.2005.00497.x. [PubMed: 16281916].

9. Bogduk N, Dreyfuss P, Baker R, Yin W, Landers M, Hammer M, et al. Complications of spinal diagnostic and treatment procedures. Pain Medicine. 2008;9(suppl_1):S11-34. 
10. Vlahos I, Chung R, Nair A, Morgan R. Dual-energy CT: vascular applications. AJR Am J Roentgenol. 2012;199(5 Suppl):S87-97. doi: 10.2214/AJR.12.9114. [PubMed: 23097172].

11. Postma AA, Das M, Stadler AA, Wildberger JE. Dual-Energy CT: What the Neuroradiologist Should Know. Curr Radiol Rep. 2015;3(5):16. doi: 10.1007/s40134-015-0097-9. [PubMed: 25815242].

12. Vogl TJ, Schulz B, Bauer RW, Stover T, Sader R, Tawfik AM. Dualenergy CT applications in head and neck imaging. AJRAm J Roentgenol. 2012;199(5 Suppl):S34-9. doi: 10.2214/AJR.12.9113. [PubMed: 23097166].

13. Postma AA, Hofman PA, Stadler AA, van Oostenbrugge RJ, Tijssen MP, Wildberger JE. Dual-energy CT of the brain and intracranial vessels. AJR Am J Roentgenol. 2012;199(5 Suppl):S26-33. doi: 10.2214/AJR.12.9115. [PubMed: 23097164].

14. Sastry VR, Manjunath KY.The course of the V1 segment of the vertebral artery. Ann Indian Acad Neurol. 2006;9(4):223.

15. Shin HY, Park JK, Park SK, Jung GS, Choi YS. Variations in Entrance of Vertebral Artery in Korean Cervical Spine: MDCT-based Analysis. Korean JPain. 2014;27(3):266-70. doi: 10.3344/kjp.2014.27.3.266. [PubMed: 25031813].

16. Hong JT, Park DK, Lee MJ, Kim SW, An HS. Anatomical variations of the vertebral artery segment in the lower cervical spine: analysis by three-dimensional computed tomography angiography. Spine (Phila Pa 1976). 2008;33(22):2422-6. doi: 10.1097/BRS.ob013e31818938d1. [PubMed: 18923317].

17. Lasjaunias P, Berenstein A, Ter Brugge KG. Clinical Vascular Anatomy and Variations. 2 ed. Berlin Heidelberg: Springer; 2001.

18. Celikyay ZR, Koner AE, Celikyay F, Deniz C, Acu B, Firat MM. Frequency and imaging findings of variations in human aortic arch anatomy based on multidetector computed tomography data. Clin Imaging. 2013;37(6):1011-9. doi: 10.1016/j.clinimag.2013.07.008. [PubMed: 23938139].
19. Berko NS, Jain VR, Godelman A, Stein EG, Ghosh S, Haramati LB. Variants and anomalies of thoracic vasculature on computed tomographic angiography in adults. J Comput Assist Tomogr. 2009;33(4):523-8. doi: 10.1097/RCT.0b013e3181888343. [PubMed: 19638843].

20. Komiyama M, Morikawa T, Nakajima H, Nishikawa M, Yasui T. High incidence of arterial dissection associated with left vertebral artery of aortic origin. Neurol Med Chir (Tokyo). 2001;41(1):8-11. [PubMed: 11218642] discussion 11-2.

21. Nalamada K, Chitravanshi N, Duffis EJ, Prestigiacomo CJ, Gandhi CD. Anomalous origin of the right vertebral artery from the right common carotid artery associated with an aberrant right subclavian artery. J Neurointerv Surg. 2013;5(5):34. doi: 10.1136/neurintsurg-2011010229. [PubMed: 22859797].

22. Duan S, He H, Lv S, Chen L. Three-dimensional CT study on the anatomy of vertebral artery at atlantoaxial and intracranial segment. Surg Radiol Anat. 2010;32(1):39-44. doi: 10.1007/s00276-009-0552-5. [PubMed: 19707709].

23. Patel MA, Caplan JM, Yang W, Colby GP, Coon AL, Tamargo RJ, et al. Arterial fenestrations and their association with cerebral aneurysms. J Clin Neurosci. 2014;21(12):2184-8. doi: 10.1016/j.jocn.2014.07.005. [PubMed: 25150765].

24. Sanders WP, Sorek PA, Mehta BA. Fenestration of intracranial arteries with special attention to associated aneurysms and other anomalies. AJNR Am J Neuroradiol. 1993;14(3):675-80. [PubMed: 8517358].

25. Yamazaki M, Koda M, Aramomi MA, Hashimoto M, Masaki Y, Okawa A. Anomalous vertebral artery at the extraosseous and intraosseous regions of the craniovertebral junction: analysis by three-dimensional computed tomography angiography. Spine (Phila Pa 1976). 2005;30(21):2452-7. [PubMed: 16261125]. 